Gut, 1963, 4, 82

\title{
Localization of intestinal bleeding using a miniature Geiger counter
}

\author{
B. MCKIBBIN AND B. W. WATSON \\ From the University Department of Surgery, Royal Infirmary, Sheffield, \\ and the Regional Medical Physics Department
}

EDITORIAL SYNOPSIS This study reports the practical problems involved in the use of a miniature Geiger counter to detect the site of bleeding into the intestine. The observations so far have been made in dogs but its possible application in man is proposed.

The difficulty in locating the site of gastrointestinal bleeding is widely recognized. Several techniques are described to assist localization in the upper reaches of the alimentary tract but very little help is available in the admittedly small group of cases in which bleeding is suspected in the small intestine, when probably more than one laparotomy has failed to reveal the source of blood loss.

The suggestion that an intraluminal Geiger counter might be used to detect extravasated red cells labelled with a radioactive isotope has been made several times, and Healey, Riggins, White, Habif, and Stewart (1961) have recorded their experience of this technique with studies in dogs. Their estimations, however, are based on a 5 to $10 \mathrm{ml} . / \mathrm{min}$. bleeding rate which, when translated for man, represents a formidable haemorrhage. Furthermore, the dose of isotope used, approximately 4 to $5 \mu \mathrm{c}$. $/ \mathrm{kg}$., would be unacceptable in clinical investigation.

An idea of the magnitude of the problem may be gained from the data of Ebaugh, Clemens, Rodnan, and Peterson (1958) who made absolute measurements of the daily blood loss from patients later proved to have lesions of the small intestine. All presented with falling haemoglobin levels and the losses ranged from $87 \mathrm{ml}$./day to as little as $18 \mathrm{ml}$./ day. To locate lesions producing this degree of bleeding an instrument capable of detecting less than $1 \mathrm{ml}$. of blood would obviously be required.

In this paper we present the results of experiments in dogs planned to elucidate some of the problems which might be expected to occur in the application of this technique to the human subject, and to formulate those conditions which would give the optimum chance of success.

\section{MATERIALS AND METHODS}

Radioactive phosphorus, $\mathbf{P}_{32}$, was the isotope selected, as in the experiments of Healey et al. (1961), on account of its absence of gamma rays whose penetrating power would mitigate against accurate localization. A maximum dose of $1 \mu \mathrm{c} . / \mathrm{kg}$. was selected as providing a not unacceptable radiation hazard when applied to the human subject.

Canine red cells, $20 \mathrm{ml}$., were labelled by incubating with from 30 to $60 \mu \mathrm{c}$. $P_{32}$ using the technique of Mollison, Robinson, and Hunter (1958). The cells were washed, resuspended in saline, and then injected intravenously into the animal, a sample having been reserved for counting.

The radioactivity of the intestine was assesse dusing a miniature halogen-quenched Geiger counter, type MX 150, commercially available from Mullard Ltd. The counter has a wall thickness of 80 to $100 \mathrm{mg} . / \mathrm{cm} .{ }^{2}$ and a maximum threshold voltage of $\mathbf{4 2 5}$ with a plateau length of 100 volts. The counter is insulated from the tissues by encasing it in a Perspex shield of $0.75 \mathrm{~mm}$. wall thickness (Fig. 1). Perspex, chosen for its insulating properties and low density, permits retention of a reasonably high efficiency for the detection of the $1.7 \mathrm{Mev} \beta$ particle from the $\mathbf{P}_{32}$. The cable supplying power to the counter enters the probe through a rubber seal to prevent the access of body fluids.

The counting was done using a normal scaler with the probe connected to the G.M. input because the presence

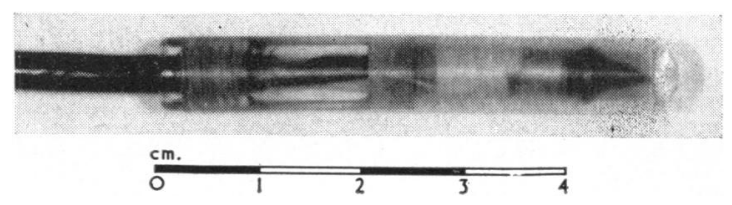

FIG. 1. The miniature counter insulated and mounted. 
.of a $10 \mathrm{M}$ resistor between the voltage supply and the probe gives added safety.

The radioactivity of samples of blood and of intestinal secretions was determined using a liquid counter (Veall, 1948). Measured blood samples were lysed with saponin and made up to $10 \mathrm{ml}$. with water before counting. Measured samples of secretions were similarly diluted to $10 \mathrm{ml}$. before being added to the liquid counter.

The experiments were carried out on mongrel dogs of body weights ranging from 15 to $20 \mathrm{~kg}$. All manipulations were done under light Nembutal anaesthesia. Three groups of experiments were performed.

\section{EXPERIMENTS}

GROUP 1: DETECTION OF SIMULATED BLEEDING LESIONS IN THE INTACT INTESTINE The cells from $20 \mathrm{ml}$. of blood labelled with approximately $20 \mu \mathrm{c}$. $\mathbf{P}_{32}$ were injected intravenously and the abdomen opened immediately, one hour, or 24 hours later. A small opening was made in the jejunum to admit the counter, extramucosal ligation of the vessels being performed to prevent contamination of the lumen with labelled blood. The background radioactivity in the lumen was then measured at various sites.

To simulate a bleeding intestinal lesion one observer, after obtaining a sample of blood by venepuncture, immediately introduced 1 to $5 \mathrm{ml}$. into the lumen by injection through the intestinal wall. The site was noted by the first observer while the second, all unknowing, attempted to locate the blood by passing the counter along the intestine. Counts were taken at 6-in. intervals or closer when blood was suspected nearby. Samples of juice from the small intestine, gastric juice, bile, and blood were taken at the same time to measure their activity.

GROUP 2: CHANGES IN BACKGROUND RADIOACTIVITY IN THE INTESTINE As a result of observations in the first series of experiments, it became clear that background radioactivity in the intestine rose considerably as soon as active cells were injected and appeared to vary with time. To study this phenomenon fully, an isolated loop of small intestine of the Thiry-Vella type was prepared in five dogs. This arrangement permitted repeated observations of intestinal radioactivity to be made for several hours after an intravenous injection of labelled cells, and daily readings were taken for up to a week afterwards. Several isolated readings were obtained one to two months after the injection.

GROUP 3: IDENTIFICATION OF THE SOURCE OF BACKGROUND RADIOACTIVITY The source of the background radioactivity was of some concern to us. Some was undoubtedly due to the radioactivity of the intestinal wall itself, and the rest to its contained secretions. A simple estimate of activity of secretions will not determine what proportion of a given counter reading is due to each moiety, since the exact geometrical arrangement of counter, intestine, and secretions is not known. To make this separation in three animals a direct fistula was made into the upper intestine as well as an isolated Thiry-Vella loop. The technique, involving the use of one side-to-side anastomosis only, is illustrated in Fig. 2. This gave a very adequate stoma even in small dogs and, although the loop was only 4 in. long, the intestinal contents did not leak since the peristaltic waves were directed away from the surface. The animals remained healthy for many months. By introducing identical counters into each loop (Fig. 3) and irrigating the isolated loop with saline, it can reasonably be assumed that the greater part of the background radioactivity in the latter is due to the intestinal wall alone, whereas secretions and products of digestion are also present in the loop giving access to the small intestine.

Serial samples of blood and gastric juice were taken, and specimens of the contents of the small intestine were obtained by catheter from the appropriate fistula.

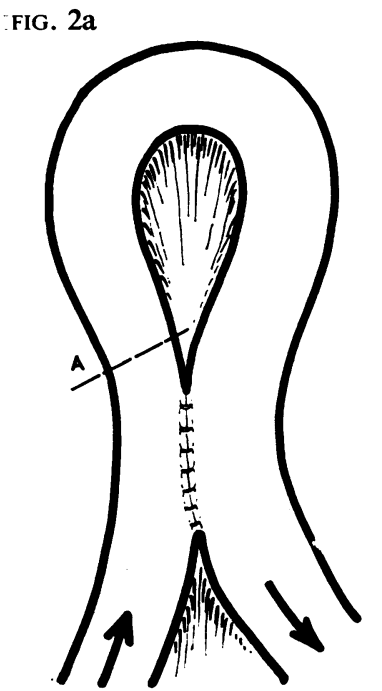

FIG. $2 b$

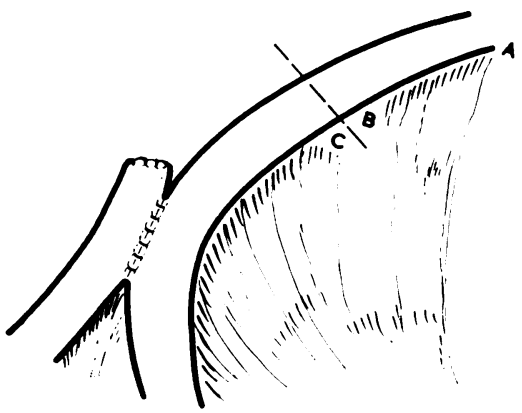

FIG. 2c

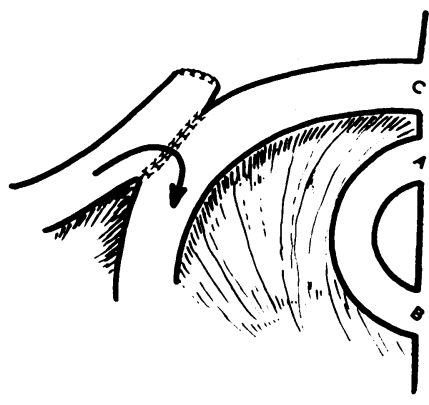

FIG. 2. Technique of construction of fistulous loops.

(a) Side-to-side anastomosis of limbs of chosen jejunal loop

(b) Loop divided at $A$ and swung up on its mesentery

(c) Portion AB used to form isolated loop. End C brought out as a separate enterostomy. 


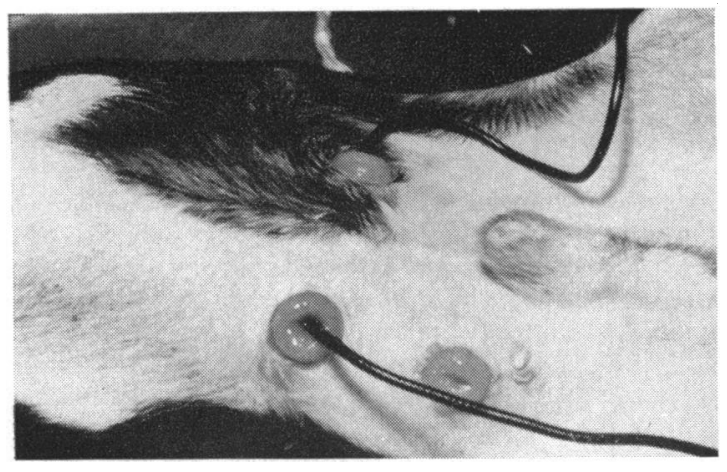

FIG. 3. Arrangement of fistulae on the dog's abdomen. Right side, counter inserted in isolated loop Left side, counter inserted into main intestine.

\section{RESULTS}

The total activity injected into the animals ranged from 18 to $40 \mu \mathrm{c}$. with an average value of $23 \mu \mathrm{c}$.

The loss of isotope from the circulation was less than $6 \%$ in one hour and less than $50 \%$ in 24 hours. Specimen curves of the pattern of decay are shown in Fig. 4. Several blood levels were taken in all the experiments and in all cases the rates of decay were comparable.

GROUP 1 EXPERIMENTS In the animals operated on immediately after injection of the isotope the background in the gut was considerable and constant $( \pm 15 \%)$ throughout the length of the small intestine. Nevertheless, it was found possible to locate as little as $1.5 \mathrm{ml}$. of intraluminal blood quite accurately, and Fig. 5 represents the result of a successful detection.

In animal studies after one to two hours, detection of $2 \mathrm{ml}$. of blood was still possible but with rather less precision; the backgrounds were generally higher and there was more variation from place to place in the intestine $( \pm 20 \%)$.

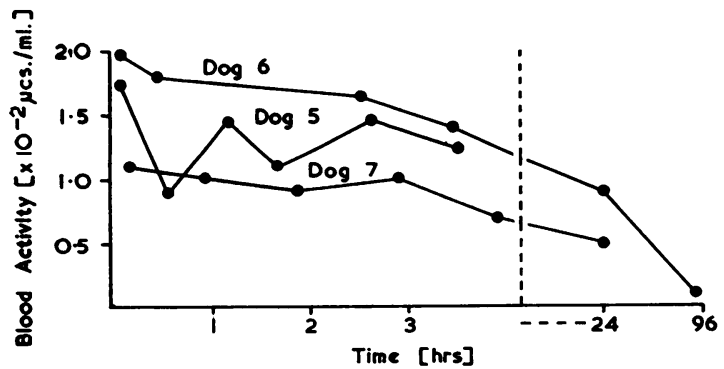

FIG. 4. Levels of blood activity after injection of cells labelled with $20 \mu c . P_{32}$ at zero time.

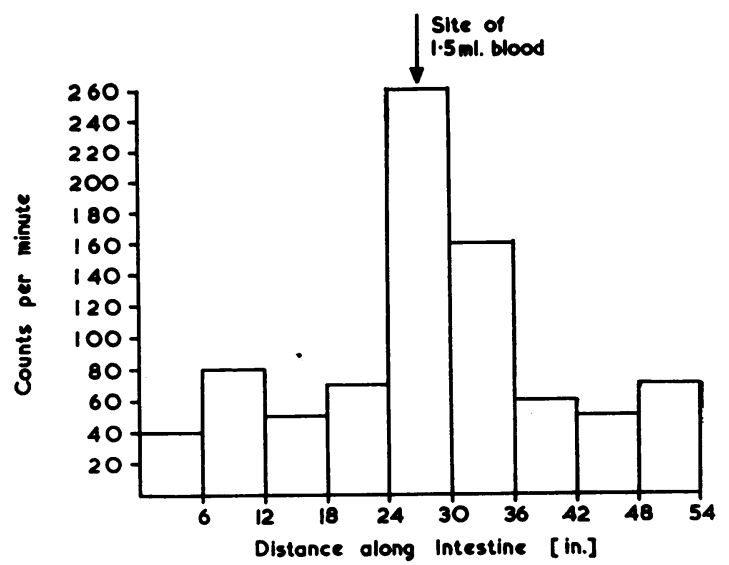

FIG. 5. Detection of $1.5 \mathrm{ml}$. of free blood in the lower jejunum.

In animals studied after 24 hours detection was not possible; the backgrounds were still higher and the blood less active. Even when relatively large volumes of blood were injected into the intestine the count was not sufficiently raised to discriminate against background.

GROUP 2 EXPERIMENTS Injection of the active material produced an immediate rise in the background radioactivity of the loop from 1 to $2 / \mathrm{min}$. to between 40 to $80 / \mathrm{min}$. and thereafter showed a progressive rise over the next 24 hours. The average rate of increase was $34 \%$ in the first hour and approximately $300 \%$ in 24 hours. Subsequent counts showed a gradual decline, but the level did not return to normal for as long as one to two months. In the earlier experiments simultaneous readings were taken at different places in the loop but, since these were found to agree closely, readings were taken at one point only in subsequent experiments. The pattern of rise is shown in Fig. 6.

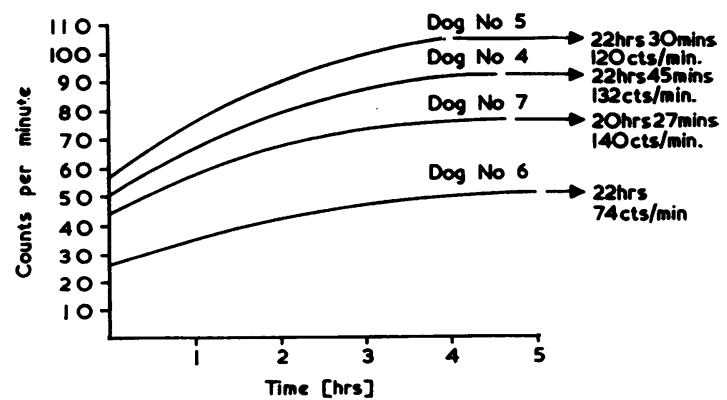

FIG. 6. Level of radioactivity in an isolated intestinal loop after the injection of $20 \mathrm{ml}$. labelled cells at zero time. The curves shown are the best fit to the experimental results allowing for the reliable error in each ordinate. 


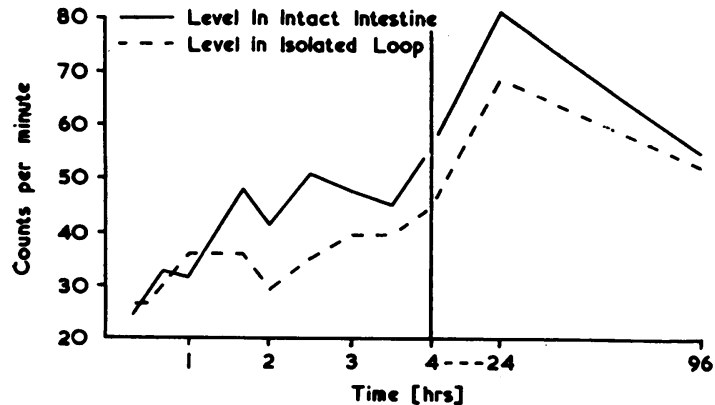

FIG. 7. Comparison of intestinal background radiation with and without secretions.

GROUP 3 EXPERIMENTS In spite of the fact that the secretions were removed by irrigation, the background radioactivity in the isolated loop was closely parallel to that in the intestine itself. In all three cases it remained almost the same for the first one and a half hours and then showed a comparative lag for the next few hours, the mean maximum discrepancy being of the order of $25 \%$. At 24 hours, and on all subsequent occasions, levels were comparable. A typical curve is given (Fig. 7).

Serial studies of secretion activity were complete only in the case of gastric juice. Secretion in the small intestine is particularly viscid in the dog and attempts to obtain multiple specimens from a catheter resulted eventually in adventitious bleeding with a consequently fallacious rise in the level of activity; some experiments had to be abandoned for this reason. Solitary specimens were therefore taken at the end of each experiment (Table I). The ratio of activity of gastric juice to that of the blood taken at the same time is given, since this is the problem in discrimination which will be put to the Geiger counter.

\section{TABLE I}

COMPARISON OF LEVELS OF RADIOACTIVITY OF BLOOD AND SMALL INTESTINAL CONTENTS IN THREE DOGS FIVE HOURS AFTER THE INTRAVENOUS INJECTION OF LABELLED RED CELLS

\begin{tabular}{rclr} 
Dog & $\begin{array}{l}\text { Activity of Blood } \\
(\mu c . / m l .)\end{array}$ & $\begin{array}{l}\text { Activity of } \\
\text { Intestinal Contents } \\
(\mu c . / m l .)\end{array}$ & Ratio \\
\hline 8 & $7 \cdot 27 \times 10^{-3}$ & $0.77 \times 10^{-3}$ & $9.4: 1$ \\
9 & $5.04 \times 10^{-3}$ & $0.5 \times 10^{-3}$ & $10.1: 1$ \\
12 & $10.81 \times 10^{-3}$ & $1.14 \times 10^{-8}$ & $9.5: 1$
\end{tabular}

The activity of gastric juice was found to be much less than that of small bowel content. At all times the activity of the blood was several hundred times more than that of the gastric juice. A typical result is given in Table II.
TABLE II

CHANGES IN RADIOACTIVITY OF BLOOD AND GASTRIC JUICE AFTER INTRAVENOUS INJECTION OF LABELLED RED CELLS

\begin{tabular}{llll}
$\begin{array}{l}\text { Time After } \\
\begin{array}{l}\text { Injection } \\
\text { (hours) }\end{array}\end{array}$ & $\begin{array}{l}\text { Activity of } \\
\text { Blood } \\
(\mu c . / m l .)\end{array}$ & $\begin{array}{l}\text { Activity of } \\
\text { Gastric Juice } \\
(\mu c . / m l .)\end{array}$ & Ratio \\
\hline \multicolumn{1}{c}{ ) } & $14.6 \times 10^{-3}$ & $0.040 \times 10^{-3}$ & $365: 1$ \\
$1 \frac{1}{2}$ & $13.4 \times 10^{-3}$ & $0.030 \times 10^{-3}$ & $446: 1$ \\
2 & $12.1 \times 10^{-3}$ & $0.035 \times 10^{-3}$ & $345: 1$ \\
3 & $11.1 \times 10^{-3}$ & $0.033 \times 10^{-3}$ & $376: 1$ \\
30 & $6.27 \times 10^{-3}$ & $0.009 \times 10^{-3}$ & $697: 1$
\end{tabular}

Isolated specimens of bile were taken in two acute experiments performed 24 hours after injection. These were found to be very active, the ratio to the activity of blood taken at the same time being $1: 2 \cdot 4$ and $1: 3$ respectively.

\section{COMMENTS ON RESULTS}

The technique of labelling used is relatively simple and although methods involving low temperature techniques and a refrigerated centrifuge give a higher percentage of isotope taken up, they are unlikely to be available in routine clinical use. However, the percentage taken up is rather irregular and it would be necessary to estimate the activity of the labelled cells before administration to patients in order to ensure correct dosage. The main disadvantage of $P_{32}$ is its relatively rapid elution from the cells, and it is important that any labelling technique shall provide as stable a bond as possible. Our results appear to be in agreement with those of Reeve and Veall (1949) where the average rate of decay was $6 \%$ in one hour giving an estimated 24-hour level of $55 \%$.

The observation that the further introduction of relatively large quantities of active blood to the intestine did not produce a proportionate increase in counting rate may have a geometrical explanation. The counter is gripped firmly by the intestinal wall and a thin film of blood only is retained between the two, the rest being disposed in such a way that it would not significantly affect the counting rate.

The results of the first group of experiments suggest that the detection of small quantities of blood within an hour or two of administering the isotope is possible but, with the passage of time, rising background and falling blood radioactivity create diffculties.

The second group of experiments confirms that there is a progressive rise in background radioactivity during the first $\mathbf{2 4}$ hours after injection. The sharp initial rise in counting rate, occurring within seconds of the isotope injection, may reasonably be interpreted as being due to labelled cells circulating 
in the intestinal wall. The subsequent increase represents the appearance of radioactive secretions in the lumen, the fixation of the isotope in the wall of the gut itself, or a combination of both factors. There is some indirect evidence that fixation does occur because detectable radioactivity is still present six weeks after injection, the blood itself having been long inactive by this time.

The third group of experiments confirms that considerable radioactivity may be detected in the absence of secretions and suggests that of any given count rate at least $75 \%$ of the responsible radioactivity must come from the intestinal wall. Thus, any attempt to improve the sensitivity of the method by reducing the background count must deal primarily with that emanating from the wall itself.

The finding of high background readings immediately after injection of labelled cells represents an essential feature of our method, since Healey $e t$ al. (1961) found no change in background after the administration of some four times the amount of isotope used by us. This discrepancy may reflect differing sensitivity of the instruments used. Although the absence of significant background is convenient in some respects, the volume of radioactive red cells required to affect the less sensitive instrument will be very much greater.

Direct studies of the radioactivity of the contents of the small bowel were not entirely satisfactory owing to the difficulty of collecting specimens free from red cells. Consequently, there is a tendency to obtain high values. Even so, it would appear that the blood is always at least 10 times as active as the secretions.

The data for gastric juice show very small amounts of radioactivity to be present. If the $\mathrm{P}_{32}$ lost from the red cells in a given time is regarded as a simple intravenous injection of this amount of free $P_{32}$, the activity of the juice may be predicted from the data of Stevens (1953), who gave intramuscular $P_{32}$ to human subjects and found the activity in gastric juice to be two or three times that of the blood at the same time. Using our data the calculated activity levels were found to be remarkably close to the measured levels, suggesting that the pathway of free phosphorus from the circulation to the stomach is the same in man as in the dog.

It can be concluded that the detection of extravasated red cells is feasible provided the investigation is carried out within an hour or two of injecting the isotope. This implies that the investigation must be employed at the time of an operation, the counter being introduced through an enterotomy. A peroral technique would have many advantages, particularly since the possibility of a telemetering device can be envisaged, but the long transit time in association with a progressively rising background count would reduce the chances of success. For this purpose it seems that an alternative device is required.

\section{DEVELOPMENT OF A MORE SENSITIVE DEVICE}

The limitations of the instrument appear to be twofold: first, the difficulty of discrimination against a level of background radioactivity which is steadily rising and, secondly, the problem of surrounding the counter, when in use, with a sufficient quantity of extravasated blood.

The counter was therefore modified by surrounding it with a copper shield $1 \mathrm{~mm}$. thick which effectively eliminates all radiation from the gut wall (Fig. 8). In addition, the wall is held away from the counter so that a greater volume of active blood can accumulate in the annular space and contribute to the count. The shield is open at both ends so that intestinal contents can pass through freely, as the device advances. The only discrimination required of the counter now is between active blood and active secretions. Since we have shown that the minimum ratio of these is $10: 1$ and the volume of the annular space is $4 \mathrm{ml}$, as little as $0.5 \mathrm{ml}$. of active blood will double the counting rate.

Unfortunately, however, this leads to a considerable increase in the size of the detector and our attempts to use it in the dog were thwarted because of the small calibre of the intestine. However, in one large dog a successful detection was possible at

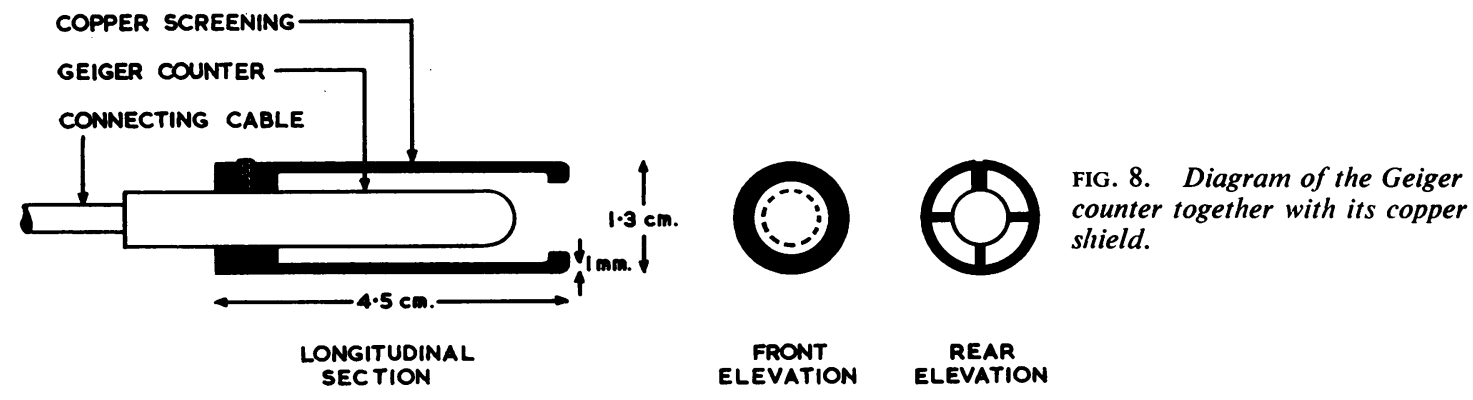


24 hours when background counts were as low as $4 / \mathrm{min}$. with the counter full of secretion. An observer was able to locate accurately $1 \mathrm{ml}$. of blood which had been previously placed in the lumen at a known site. Counts as high as $100 / \mathrm{min}$. were recorded when some of the blood entered the counter.

The size of the screened counter, which in its present form would be beyond the power of most subjects to swallow, presents a problem. However, it is hoped in the near future to use a semi-conductor detector instead of the Geiger counter. These detectors are much smaller $(5 \mathrm{~mm} . \times 5 \mathrm{~mm}$. $)$ and work at a lower voltage (less than 100 volts). Not only would the overall size of the device be reduced but, because of the more modest power demands, the prospect of incorporating the whole in a telemetering capsule becomes more realistic.

\section{CLINICAL APPLICATIONS}

It is felt that enough basic information is now available to enable an attempt to be made to locate a bleeding lesion in the human subject with some prospect of success.

Peroral techniques must be set aside pending the development of a smaller device. Consequently, the counter must be passed through the intestine at operation. In any technique of this sort the timing of the investigation relative to the administration of the isotope is critical; although, as time passes, more blood will be available for detection, yet the level of blood activity is falling as the level of background radio-activity in the gut is rising. The precise time will depend on an estimate of the degree of bleeding and obviously the greater this is the shorter may be the time between injection and operation. In general our results suggest that an interval of one hour would represent a reasonable compromise and the following technique is suggested:

Blood, $50 \mathrm{ml}$., is withdrawn from the subject and labelled with $\mathrm{P}_{32}$ using the technique described above. A suspension of labelled cells, containing approximately $1 \mu \mathrm{c}$. $\mathrm{P}_{32} / \mathrm{kg}$. body weight, is injected intravenously. One hour later the abdomen is opened and, failing the clinical detection of a lesion, a small opening is made in the duodeno-jejunal flexure. Great care must be taken to avoid the contamination of the lumen with blood and the technique of extramucosal ligation is suggested. Both counters would be passed the full length of the small intestine, for although the screened counter is capable of detecting smaller quantities of free blood yet it can take no account of clot or even of a vascular lesion itself. Counts over a one-minute period should be taken at 6-in. intervals or closer when the blood is thought to be near, as indicated by at least a doubling of the count rate. The incorporation of an audio signal makes the appreciation of a small change in count rate more apparent.

We are indebted to Professor A. W. Kay for much helpful advice and encouragement with this work and to Mr. F. Naylor for invaluable technical assistance. One of us (B. McKibbin) is in receipt of a full-time grant from the Medical Research Council.

\section{REFERENCES}

Ebaugh, F. G., Clemens, T., Rodnan, G., and Peterson, R. E. (1958). Quantitative measurement of gastrointestinal blood loss. Amer. J. Med., 25, 169-181.

Healey, W. V., Riggins, R. C., White, R. L., Habif, D. V., and Stewart, W. B. (1961). An experimental method for localizing the source of intestinal bleeding. Surg. Gynec. Obstet., 112, 285-288.

Mollison, P. L., Robinson, M. A., and Hunter, D. A. (1958). Improved method of labelling red cells with radioactive phosphorus. Lancet, 1, 766-769.

Reeve, E. B., and Veall, N. (1949). A simplified method for the determination of circulating red-cell volume with radioactive phosphorus. J. Physiol. (Lond.), 108, 12-23.

Stevens, J. (1953). The secretion of radioactive phosphorus in saliva and gastric juice in man. Clin. Sci., 12, 375-383.

Veall, N. (1948). A Geiger-Muller counter for measuring the betaray activity of liquids, and its application to medical tracer experiments. Brit. J. Radiol., 21, 347-351. 